\title{
MIROIR, MIROIR? COMMENT PUIS-JE ME RATTRAPER POUR ENGAGER DES JEUNES EN GÉNIE?
}

\author{
Janelle Fournier \\ Université d'Ottawa \\ jfour055@uottawa.ca
}

\begin{abstract}
Résumé - En Amérique du Nord, le besoin d'une relève d'élèves qui souhaite poursuivre des carrières en STIM est criant [3, 38, 48]. Les gouvernements de plusieurs pays, incluant le Canada, ont donc réalisé l'importance de l'enseignement en STIM et il est devenu évident qu'il y a un manque de compréhension de ce dernier [29].

Afin de faire avancer l'enseignement du génie dans les cours de sciences et mathématiques, nous avons mené une auto-ethnographie sur notre expérience comme enseignante de sciences et de mathématiques au palier secondaire. Nous sommes fières d'avoir été capables d'engager des élèves d'une diversité de genres aux activités d'apprentissage en STIM. Pourtant, nos activités ont été concentrées sur les sciences, les technologies et les mathématiques plutôt que sur l'ingénierie; une pratique qui doit changer afin d'inciter plus d'élèves à se diriger dans cette profession [42].
\end{abstract}

Mots-clés : Enseignement au secondaire, Ingénierie, STIM, Auto-ethnographie, Pratique pédagogique, Transdisciplinarité, Interdisciplinarité

\section{INTRODUCTION}

Le gouvernement du Canada [20] proclame que « pendant la prochaine décennie, les économies avancées devront colmater les lourds déficits budgétaires provenant de la crise économique actuelle, s'adapter aux récentes innovations, réduire les répercussions des changements climatiques et affronter la concurrence féroce des nouvelles économies » [paragr. 2]. Selon ce cadre législatif, les innovations en sciences et technologies peuvent contribuer à résoudre ces nombreux problèmes [20]. Cependant, il est stipulé qu'afin d'être capables de résoudre ces grands problèmes mondiaux, nous avons besoin de personnes qualifiées en sciences, technologies, ingénierie et mathématiques (STIM) [47].

Par ce fait même, la demande pour plus de personnes qualifiées en STIM continue à croître. En fait, le département de commerce des États-Unis remarque qu'entre les années 2008 et 2018, les emplois en STIM montrent un pourcentage de croissance plus élevé (dont
17 pourcent) que les emplois qui n'impliquent pas de STIM (9,8 pourcent) [33]. Il est prévu qu'en 2020 , il y aura deux millions d'emplois à combler en informatique, en mathématiques, en architecture et en ingénierie [45]. Plus spécifiquement, plus de 339000 postes seront vacants au cours des quatre prochaines années dans les disciplines du génie civil, mécanique, industriel et électrique [51].

Malgré des conditions avantageuses, il n'y a toujours pas suffisamment de jeunes personnes, notamment de jeunes femmes, intéressées à poursuivre des carrières en STIM pour combler tous ces postes [43]. Alors que 36 pourcent des garçons sont intéressés aux carrières en STIM, pour les filles, ce pourcentage diminue à 11 pourcent [13]. Selon Kimmel, Carpinelli, Burr-Alexander et Rockland [31], une des raisons pour lesquelles il y a peu d'intérêt pour les carrières en STIM, particulièrement en génie, est que les élèves ne sont pas adéquatement exposés à des situations d'apprentissage pertinentes dans les cours de sciences et de mathématiques au palier secondaire.

Par conséquent, nous avons décidé de mener une autoethnographie sur notre temps en enseignement des sciences et des mathématiques dans une école de langue française en Ontario. L'auto-ethnographie nous a permis d'évaluer notre propre enseignement des STIM (plus précisément notre enseignement de l'ingénierie) et nous a permis de comparer nos expériences professionnelles à la recherche en éducation [18].

\section{PROBLÉMATIQUE}

À cause du besoin d'une relève de jeunes personnes qui désirent poursuivre des études et des carrières en STIM [3, 38, 48], les gouvernements de plusieurs pays, incluant le Canada et les États-Unis, ont donc réalisé l'importance de l'éducation en STIM. L'enseignement des STIM est une approche pédagogique qui demande d'enseigner les sciences, la technologie, l'ingénierie et les mathématiques de façon interdisciplinaire ou transdisciplinaire. Plutôt que d'enseigner des concepts dans des cours séparés, les STIM incorporent de façon fluide, interactive et innovante les quatre domaines $[6,8]$. 
C'est un processus d'enseignement et d'apprentissage qui donne l'occasion aux élèves de comprendre de façon plus réaliste et complète ce qui se passe dans le monde et offre une occasion qu'ils soient au centre de leur apprentissage [26]. Des problèmes sont tirés du monde réel et les élèves ont la tâche d'y trouver des solutions [6]. Pour ce faire, ils vont vivre des expériences, collaborer, découvrir, créer et bâtir [26]. L'enseignement des STIM tente d'améliorer les compétences du $21^{\mathrm{e}}$ siècle, dont la pensée critique, la résolution de problèmes, l'innovation, la créativité, les connaissances de soi, l'autoapprentissage, la collaboration et la communication [15], mais aussi de comprendre comment les sciences, la technologie, l'ingénierie et les mathématiques influent sur le monde $[8,26]$.

Afin d'accorder une plus grande importance à l'éducation des STIM, le gouvernement du Canada a lancé plusieurs initiatives pour promouvoir les carrières en STIM. En 2009, il a contribué au financement de Youth in Science, Technology, Engineering and Mathematics et au mois de février 2017, la campagne de sensibilisation Optez pour les sciences a été lancée pour encourager les jeunes filles à s'intéresser aux STIM [17, 28]. Le site web pour la campagne Optez pour les sciences, offre des ressources aux parents et au personnel enseignant afin de mener des activités d'apprentissage amusantes et intéressantes qui susciteront les intérêts des jeunes en STIM [28]. En 2017, Canada 2067 [11] fut aussi initié pour introduire « les Canadiens dans une nouvelle vision de l'apprentissage des STIM » [paragr. 1]. Canada 2067 a comme objectifs que tous les élèves soient inscrits dans un cours supérieur en STIM; que le pourcentage d'étudiants inscrits dans des programmes postsecondaires en STIM augmente de 20 à 25 pourcent; que le nombre d'enseignantes et d'enseignants avec des spécialisations en sciences ou en mathématiques augmente radicalement; et que les gouvernements investissent un pourcent des budgets dans la recherche en STIM afin de soutenir la réalisation de ces objectifs [11].

Bien que les initiatives gouvernementales offrent de l'appui à l'enseignement des STIM, ces dernières sont aussi incluses dans le programme-cadre de l'Ontario. Le programme-cadre de sciences et de technologies de la première à la huitième année a trois objectifs : lier les sciences et les technologies à la société et à l'environnement; développer les habiletés et les stratégies nécessaires à la résolution de problèmes technologiques qui mènent à des enquêtes scientifiques; et comprendre les concepts de base de sciences et de la technologie [40]. Le but du programme-cadre de mathématiques est que les élèves comprennent les mathématiques, développent une pensée critique et créative, utilisent un langage mathématique, appliquent leurs connaissances à différents contextes et fassent des liens avec la vie quotidienne [40]. Quoique les programmes-cadres ne fassent pas explicitement mention de l'enseignement de l'ingénierie [17], ils l'évoquent implicitement dans les sections qui abordent le rapprochement entre la culture scientifique et technologique et l'environnement [40]. Cela est sur le point de changer, car au mois de mars 2019, le gouvernement de l'Ontario a annoncé que l'ingénierie (codage) sera obligatoire dans les cours d'études informatiques [41].

Actuellement, malgré les nombreux efforts et initiatives en éducation, l'enseignement des STIM en Amérique du Nord se concentre sur les sciences, les technologies et les mathématiques, plutôt que sur l'ingénierie [42]. Bull, Knezek et Gibson [9] considèrent que ce phénomène doit changer, car l'ingénierie agit comme le fil connecteur pour les sciences, la technologie et les mathématiques. Turkle [46], une professeure de sciences, technologie et société au Massachusetts Institute of Technology explique que les élèves s'intéressent aux sciences, car ils sont passionnés par l'ingénierie des objets scientifiques. À titre d'exemple, elle mentionne que les élèves sont fascinés par la construction de châteaux de sable et qu'ils sont curieux du fonctionnement d'une unité d'air climatisé, mais qu'ils ne réalisent pas à quel point des objets scientifiques impliquent plus que la science. Afin de concevoir ces objets, il est nécessaire d'avoir une compréhension des sciences, des technologies, des mathématiques et bien sûr, de l'ingénierie. Puisque l'enseignement des STIM se concentre moins sur l'ingénierie, les élèves sont moins exposés à des activités pédagogiques dans ce domaine. Ce nombre limité d'occasions d'apprentissage du génie contribue à la pénurie de jeunes personnes, spécifiquement de jeunes femmes, en ingénierie [42].

Afin de faire avancer l'enseignement du génie dans les cours de sciences et mathématiques de la maternelle à la douzième année, nous avons décidé (avant tout) de regarder dans le miroir pour réfléchir à nos années d'expérience en éducation par l'entremise d'une autoethnographie. Nous évaluons les expériences qui ont mené à un enseignement interdisciplinaire et éventuellement transdisciplinaire des STIM et notre état de préparation face à l'enseignement de l'ingénierie. En effet, l'état de préparation est l'un des principaux facteurs qui mènent à l'enseignement de l'ingénierie et qui affectent la motivation, l'engagement et la persévérance $\mathrm{du}$ corps enseignant [50]. L'état de préparation fait référence à nos perceptions actuelles du domaine d'ingénierie, nos connaissances du contenu en génie et notre sentiment d'efficacité personnelle (SEP). Ces trois attributs influencent l'enseignement des concepts du génie qui se fait dans les cours de sciences et mathématiques.

\section{CADRE THÉORIQUE}

Lent, Brown et Hackett [35] utilisent la théorie sociale cognitive de Bandura pour comprendre ce qui influe sur le développement de la carrière. Dans leur théorie sociale cognitive de l'orientation scolaire et professionnelle 
(TSCOSP), l'accent est placé sur les variables cognitives de la personne, dont le SEP, les attentes de résultats, les objectifs personnels, et la façon dont ces variables interagissent avec les caractéristiques personnelles et les potentialités offertes par le contexte (dont, les perceptions) pour prédire les choix académiques et professionnels [14].

Le SEP est la croyance de la personne en sa capacité à réaliser une activité [5]. Le jugement d'une personne est affecté par les attentes de résultats qui, par la suite, incitent un comportement spécifique par exemple, le gain d'argent, la satisfaction personnelle ou l'approbation sociale [22]. Les objectifs personnels sont les intentions d'une personne à s'engager dans une activité afin d'atteindre un but particulier [34].

Selon Lent [34], les caractéristiques personnelles, telles que le genre, l'ethnicité, les années d'expérience, le domaine et le palier d'enseignement et les potentialités offertes par le contexte, telles que les perceptions, contribuent aux expériences et aux apprentissages (ou connaissances du contenu). Ces expériences et apprentissages développent ensuite le SEP et les attentes de résultats. Ces derniers ont ultérieurement une influence sur les intérêts professionnels. Pour Lent [34], Viau [50] et Yoon, Diefes-Dux et Strobel [54], les gens s'intéressent plus aux activités professionnelles et démontrent une motivation, un engagement et une persévérance envers ses activités, quand ils se trouvent compétents et quand ils s'attendent à des résultats positifs. Une fois intéressé à une activité, comme l'enseignement des STIM ou du génie, le personnel enseignant fait un choix de buts et d'actions. D'après ces actions, il y a soit un succès ou un échec qui, lors des efforts subséquents, influent sur le SEP et l'atteinte de résultats [36]. Lent [34] ajoute qu'il existe des influences contextuelles relatives aux choix (comme les collègues de travail et l'opportunité de formations) qui affectent les choix de buts et d'actions. "De manière générale, l'environnement à travers les ressources sociales et matérielles qu'il [l'enseignant] met à disposition va déterminer l'étendue des choix professionnels et des opportunités de développement » [Loisy 66].

La TSCOSP aide à faire comprendre le choix d'actions du personnel enseignant à s'engager dans des activités de STIM et du génie. La théorie montre que le choix d'actions est un processus qui découle en premier lieu des caractéristiques personnelles et des perceptions, en deuxième lieu des connaissances du contenu et en dernier lieu, du SEP de l'individu.

\section{IMPORTANCE DE L'ENSEIGNEMENT DU GÉNIE}

Actuellement, les avancées technologiques influencent beaucoup la façon dont les personnes vivent et interagissent entre elles. Il est donc important que les citoyens aient des connaissances de base en technologie.
Selon le National Research Council, la technologie se définit comme une modification du monde naturel pour répondre aux besoins ou aux vouloirs humains, tandis que l'ingénierie est une approche systématique et souvent itérative de la conception d'objets pour répondre aux besoins et aux désirs des humains [42]. Essentiellement, l'ingénierie joue un rôle crucial dans la conception de technologies et permet de faire des liens entre les sciences et les mathématiques, et le monde réel [9, 39].

L'Association internationale des enseignants de technologie et d'ingénierie considère que les élèves devraient, dès l'école primaire, développer une culture technologique comprenant une compréhension plus large du développement des technologies, évaluer les effets de la technologie et comprendre comment la technologie se rapporte aux autres domaines d'étude et affecte la société ; tout cela dans le but de former des citoyens qui sont habiles en technologie. Pour préparer des citoyens qui sont habiles en technologie, les élèves de la maternelle à la douzième année doivent développer une compréhension des concepts d'ingénierie [22].

En plus de former des citoyens habiles en technologie, les élèves exposés à l'enseignement de l'ingénierie ont une meilleure compréhension en mathématiques et en sciences et sont plus à l'aise à résoudre des problèmes difficiles en utilisant une pensée critique et créative [25, $38,52]$.

Le National Academy of Engineering et le National Research Council [42] ajoutent que les élèves habiles en technologie ont une meilleure connaissance des diverses carrières en génie et des tâches des ingénieurs. La recherche menée par Hammack, Ivey, Utley et High [24] a montré que les élèves en sixième année qui participent à un camp d'été d'ingénierie d'une durée d'une semaine ont une meilleure compréhension de la technologie et des tâches des ingénieurs. La probabilité qu'ils entreprennent des carrières en génie est aussi plus élevée quand il y a eu un enseignement dans ce domaine. L'enseignement d'ingénierie est même lié à une meilleure assiduité scolaire [42]. De plus, un bon enseignement (formel ou informel) peut aussi inciter plus de filles à s'intéresser aux programmes d'ingénierie [49]. Ferreira [19] a effectué une étude de cas pour déterminer l'impact d'un programme parascolaire auprès de 18 filles à l'intermédiaire. L'étude a souligné que 14 des filles voulaient poursuivre le génie après avoir participé au programme. Une autre recherche menée par Anderson, Gilbridge et Bajaj [1] met en évidence que 80 pourcent des filles du secondaire qui ont participé à un camp d'été en ingénierie $(\mathrm{n}=350)$ ont démontré plus d'intérêt envers la profession d'ingénierie. L'objectif du camp était d'initier des filles à poursuivre des carrières en ingénierie, en les exposant aux défis et aux apports de ce domaine par l'entremise d'activités et de discussions. Il fut mené par des professionnelles en génie et en sciences, ainsi que des étudiantes dans ce même domaine. D'après les 
auteurs, 30 pourcent des participantes ont poursuivi des carrières en génie après le secondaire.

\section{MÉTHODOLOGIE}

Cette étude porte un regard sur nos expériences vécues en enseignement au palier secondaire. Après avoir passé neuf années dans cette profession, nous avons commencé nos études au doctorat et avons réfléchi à notre expérience en tant qu'enseignantes. Par conséquent, nous avons décidé de mener une auto-ethnographie qui combine des caractéristiques de l'autobiographie et de l'ethnographie [18]. Selon Ellis, Adams et Bochner [18], le but de 1'autoethnographie est d'offrir un regard sur notre expérience culturelle, dans notre cas, l'enseignement des sciences et des mathématiques. Il s'agit de réfléchir à nos expériences passées et de déterminer ce que nous aurions pu améliorer quant à notre enseignement des STIM. Il nous est aussi important d'évaluer notre état de préparation à enseigner le génie. Il ne s'agit pas de faire un sommaire de nos expériences, mais plutôt de porter un regard critique sur ces expériences [32] afin d'en apprendre de celles-ci. Finalement, l'auto-ethnographie permet de faire des liens entre nos expériences professionnelles et la recherche sur l'enseignement des STIM, et ce, tout en visant l'avancement du dossier de l'enseignement du génie [18].

Dans une auto-ethnographie, la chercheuse est le sujet de l'étude, donc l'étude permet de connaitre un point de vue qui serait autrement indisponible [32]. Au lieu de penser que mon rôle est plutôt externe (une personne qui observe l'enseignement en salle de classe), mes réflexions personnelles nous ont permis de percevoir notre rôle comme étant une personne à l'interne qui est acceptée dans son champ de spécialité et qui connait bien le contexte [16].

Cette approche est une méthodologie appropriée puisqu'il nous manque la voix des enseignants en Ontario face à l'enseignement du génie. Nous ne prétendons pas que notre point de vue remplace ceux de tous les enseignants en Ontario, mais plutôt que notre expérience pourrait être conforme à d'autres enseignants. Ceci est important, car il n'y a pas d'études qui peuvent nous aider à connaitre leurs expériences et leur état de préparation à entreprendre cet enseignement.

Nous sommes choyés, car nous pouvons combiner notre travail professionnel avec notre recherche. Ceci pourrait cependant être perçu comme étant problématique pour les lecteurs à cause des biais et du manque d'objectivité. Une limite de cette étude est qu'il inclut la perspective d'une seule personne. Ceci étant dit, le cas d'une personne peut être utile pour nos apprentissages et peut nous informer d'un plus gros problème [53]. L'avantage d'une recherche à la première personne est qu'elle peut donner une «compréhension nuancée des événements significatifs et des contextes sociaux utilisés » [Knapp 255]. L'auto-ethnographie est décrite comme un chemin de collaboration entre l'auteure et le lecteur [32].

\subsection{Contexte de l'étude}

L'étude fait référence à notre temps en enseignement dans une école secondaire de langue française en Ontario. L'école se situe dans le sud de l'Ontario où il y avait environ 550 élèves et 46 membres du personnel. Les administrateurs étaient ouverts au changement des approches pédagogiques qui assure le bien-être des élèves et l'apprentissage de ceux-ci. Nous faisions partie de communautés d'apprentissage professionnelles et avons accès à une accompagnatrice en mathématiques et une conseillère pédagogique en sciences et mathématiques.

\subsection{Collecte et analyse de données}

Nos données sont des réflexions personnelles basées sur nos expériences du passé. Nous avons pris note des événements qui ont marqué l'évolution de notre enseignement et avons tenté de faire ressortir les points importants qui ont changé notre philosophie de l'enseignement ou nos pratiques pédagogiques. Pour ce faire, nous avons noté nos expériences en enseignement. Ensuite, nous avons surligné tous les points qui ont incité un changement dans notre enseignement des STIM. En ce qui concerne l'état de préparation, nous avons noté nos perceptions des ingénieurs et de l'ingénierie, nos connaissances du processus de design et notre sentiment d'efficacité personnelle face à l'enseignement du génie. Une question qu'on s'est posée est : est-ce que nous nous sentions à l'aise à concevoir des occasions d'apprentissage en génie?

\section{RÉSULTATS}

Les résultats de notre auto-ethnographie sont divisés en deux parties : les moments qui ont fait évoluer notre enseignement des STIM et notre état de préparation face à l'enseignement du génie.

\subsection{Moments qui ont changé notre enseignement}

Les moments de changement se sont passés plus tard dans notre carrière en enseignement (après environ 4 ans). Ils furent quand nous n'étions pas satisfaites avec le niveau de réussite des élèves ou nous avions remarqué que les élèves éprouvaient les mêmes difficultés d'année en année. À titre d'exemple, l'étude des projectiles en physique était difficile pour les élèves à comprendre. Le programme-cadre demandait que les élèves déterminent le temps que passe un objet en projectile avant de toucher le sol. Pour ce faire, les élèves devaient utiliser la formule quadratique qu'ils ont apprise en mathématiques. Les élèves avaient beaucoup de difficulté à résoudre ce problème, parce qu'ils ne faisaient pas de liens entre les processus mathématiques et les concepts scientifiques. Il 
est devenu évident qu'il devait y avoir plus de liens explicites entre les sciences et les mathématiques. Cette observation nous a menés à changer notre façon d'enseigner et de se diriger vers une approche interdisciplinaire.

Un deuxième changement a eu lieu quand certains membres du personnel enseignant des départements des sciences et des mathématiques se sont concertés pour enseigner des élèves qui éprouvaient des difficultés dans un cours de mathématiques en $9^{\mathrm{e}}$ année. Puisque les élèves manquaient de motivation et avaient une crainte réelle des mathématiques, nous avions décidé de travailler avec la conseillère pédagogique et l'accompagnatrice en mathématiques dans le but de concevoir des activités d'apprentissage qui traitaient du vécu des élèves et des problèmes authentiques de la société. En observant l'engagement de tous les élèves (garçons et filles) et en évaluant leur réussite, nous avions réalisé l'importance de l'enseignement transdisciplinaire des STIM. Ceci a encore une fois fait évoluer notre enseignement en mathématiques et en sciences. Au fil des années, ces occasions d'apprentissage interdisciplinaire et transdisciplinaire de nature variée (par ex. la création d'un parfum en chimie ou la fabrication d'une montagne russe en physique et en mathématiques), ainsi que les attentes élevées que nous avions pour tous les élèves, ont incité une diversité de genres d'élèves à se diriger dans le domaine des STIM.

Bien qu'il y ait eu une évolution de notre enseignement, l'accent fut toujours placé sur les sciences, les mathématiques et même les technologies. Encore une fois, l'ingénierie ne faisait pas partie des discussions ou de l'enseignement. Ceci nous a amenés à faire une réflexion sur notre état de préparation face à cet enseignement.

\section{2. État de préparation}

Nous avions réalisé que pendant le temps que nous enseignions, nous n'étions pas prêts à entreprendre un enseignement du génie. Nous avions une perception positive du travail des ingénieurs et ne détenions pas de stéréotypes face à la profession, et nous respections la profession d'ingénierie et leur apport à la société. Cela étant dit, nos connaissances du travail des ingénieurs étaient limitées. Puisque la portée de leur travail est tellement vaste, il était difficile à établir une liste de leurs rôles et responsabilités, car chaque discipline différait un de l'autre (ex. les tâches journalières d'un ingénieur biomédical diffèrent considérablement du travail d'un ingénieur civil). Bien que nous réalisions maintenant que les ingénieurs entreprennent le processus de design, ce concept ne nous était pas connu pendant que nous enseignions. Nous avions donc des connaissances de contenu incomplètes face au processus de design alors notre sentiment d'efficacité personnelle n'était pas élevé.
On ne se croyait pas habile à entreprendre des activités d'ingénierie.

\section{DISCUSSION}

En regardant dans le miroir, nous avons réfléchi à nos années d'expérience acquises en éducation. Nous sommes fières d'avoir été capables d'engager des élèves d'une diversité de genres aux activités d'apprentissage en STIM. Nous réalisons que l'enseignement des STIM de façon interdisciplinaire ou transdisciplinaire demande une certaine maitrise du curriculum, une expérience en enseignement et une persévérance. L'enseignante doit être capable de faire les liens entre les disciplines; ce qui n'est pas toujours évident pour des enseignants débutants. Il n'est pas raisonnable de s'attendre à ce que le personnel enseignant débutant se lance immédiatement dans la transdisciplinarité ou l'interdisciplinarité. Le personnel enseignant chevronné peut cependant se fixer des objectifs de rendre son enseignement plus centré sur les apprenants et qui tente de résoudre des problèmes authentiques de la société; ce qui s'intègre bien avec la profession d'ingénierie qui travaille à faire avancer la société.

En ce qui a trait à l'enseignement du génie, nous avons remarqué que notre auto-ethnographie ressemble beaucoup aux résultats d'études américaines. Selon les articles de Boesdorfer [4] et Hsu, Purzer et Cardella [25], les enseignants semblent avoir des perceptions positives de l'ingénierie. Ils perçoivent l'ingénierie comme étant un domaine créatif et excitant qui exige une pensée critique [4]. De plus, les ingénieurs sont perçus comme étant des professionnels ayant des compétences sociales, de bonnes habiletés de communication orale et écrite, des habiletés en mathématiques et en sciences et ils ont un impact positif sur la société [25]. Bien que les auteurs disent que le personnel enseignant n'entretient généralement pas de stéréotypes de la profession d'ingénierie, qu'il comprend ce qu'est l'ingénierie et détient des perceptions plus justes que celui du public, ces dernières sont toujours inexactes. En pensant que les ingénieurs réparent des objets et font un travail semblable aux scientifiques, nous constatons qu'ils détiennent toujours des conceptions naïves de l'ingénierie. Pour les connaissances du contenu, le personnel enseignant a de la difficulté à définir le processus de design $[2,4,23,25]$. D'après les articles analysés, nous concluons que le personnel enseignant n'est pas au courant des méthodes à suivre pour incorporer le génie dans les cours de sciences et mathématiques à cause d'un manque de connaissances des étapes clés du processus de design.

Le processus de design est utilisé par les ingénieurs pour tenter de résoudre un problème à multiples solutions. Ces étapes sont: définir et comprendre le problème; explorer ce qui a déjà été fait et recueillir toutes informations pertinentes; utiliser sa créativité pour 
concevoir des idées qui vont résoudre le problème; construire un prototype fonctionnel; tester le prototype sous de vraies conditions; et évaluer et éditer le prototype pour déterminer s'il s'agit de la meilleure solution au problème. Si ce n'est pas le cas, le processus recommence [30].

Ce processus peut être utilisé en salle de classe, mais la majorité du personnel enseignant ne se sent pas à l'aise avec l'enseignement des STIM ou du génie. Banilower et coll. [2] et Hammack et Ivey [23] ont noté qu'en moyenne 95,5 pourcent du personnel enseignant possède un niveau faible de SEP et ceci peut être attribué à un manque d'expériences en génie. Carberry, Lee et Ohland [12] disent que le SEP en génie s'améliore quand le nombre d'expériences en génie augmente. Alors, il est probable que le niveau de familiarité du génie et les connaissances du processus de design affectent le SEP du personnel enseignant; ce qui appuie la TSCOSP [23].

Nous constatons qu'actuellement le personnel enseignant n'est pas confiant dans ses connaissances en génie ni dans ses capacités à enseigner ce dernier. Essentiellement, le corps enseignant n'est pas prêt à aborder un enseignement de l'ingénierie. Ce constat s'aligne bien avec nos réflexions personnelles.

Nous devons avouer qu'après avoir fait de la recherche sur l'enseignement du génie, la profession d'ingénierie et le processus de design utilisé par les ingénieurs, notre état de préparation a changé substantiellement et nous nous sentions prêts à aborder cet enseignement. Nous nous sommes familiarisés avec le processus de design et à l'heure actuelle, nous sommes d'avis qu'il peut aisément être introduits à la salle de classe de sciences et mathématiques. Pour ce faire, nous proposons que des concepts scientifiques et mathématiques soient abordés pendant le processus de design, précisément lorsque les élèves explorent des solutions possibles au problème [21].

Ceci étant dit, le personnel enseignant nécessite une formation continue afin d'appréhender le processus de design en génie et de concevoir des activités qui impliquent ce processus. En ce qui concerne la formation à l'enseignement, le génie devrait faire partie des cours de didactiques des sciences et mathématiques ou, mieux encore, il devrait y avoir un cours propre à l'enseignement des STIM. De fait, en 2005, aux États-Unis, l'école Virginia Tech a commencé à offrir un tel cours afin de permettre au personnel d'entreprendre l'interdisciplinarité ou la transdisciplinarité en STIM. Entre autres, ce cours aide les futurs enseignants à établir les liens qui existent entre les disciplines des STIM [44].

\section{CONCLUSION}

Puisqu'il y a un besoin de personnes qualifiées en STIM [38] et que l'ingénierie agit comme un fil conducteur pour ces matières [9], les élèves tireraient profit d'un enseignement du génie.

L'intégration des principes de génie par l'entremise de résolutions de problèmes avec le processus de design peut engager davantage les élèves dans les cours de sciences et mathématiques, améliorer leurs compétences du $21^{\mathrm{e}}$ siècle, et susciter chez les élèves, spécialement les filles, un intérêt à poursuivre une carrière en génie (ou en STIM) $[10,31]$. Par contre, à l'heure actuelle, l'enseignement de l'ingénierie n'est pas suffisant, car le nombre d'élèves intéressés à ces carrières n'augmente pas assez pour contribuer à l'avancement de notre société [10].

C'est pour cela qu'il est important de considérer les expériences dans l'enseignement des STIM et l'état de préparation du personnel enseignant pour entreprendre un enseignement du génie. Ce sont les enseignants qui peuvent avoir un grand impact sur le développement des habiletés et susciter l'intérêt des élèves en génie [7]. L'information recueillie à partir de notre autoethnographie nous aide à éclairer cet état de préparation.

En fait, nos résultats montrent que nous avons une perception positive de la profession d'ingénierie, nous reconnaissons l'importance de l'enseignement des STIM de façon transdisciplinaire et du génie, mais nous avons toujours des lacunes face à la compréhension du travail des ingénieurs. De plus, nous n'avions pas les connaissances en génie pour entreprendre cet enseignement, car nous n'avions pas suivi de cours en génie au postsecondaire. Ce constat est conforme aux résultats de Banilower et coll. [2] lorsqu'ils déclarent que seulement 7,33 pourcent du personnel enseignant a suivi des cours postsecondaires en génie. Afin d'améliorer notre SEP face à cet enseignement, il faut que nous obtenions des connaissances plus approfondies du domaine de génie, dont le processus de design. Ce niveau faible du SEP affecte nos habiletés à enseigner le génie [36].

Quoique nous comprenons maintenant la valeur de l'enseignement du génie, lors de notre expérience vécue nous n'avions pas les connaissances du processus de design et n'avions pas un SEP élevé, donc le génie n'était pas incorporé en salle de classe. Cela nous a amenés à la réalisation qu'il serait profitable d'avoir des occasions de développement professionnel dans le domaine du génie. Bien que nous ne soyons qu'une enseignante, nous sommes d'avis que plusieurs autres enseignants pourraient profiter d'une formation continue dans l'enseignement du génie.

Les occasions de développement professionnel devraient être axées sur la formation du personnel enseignant quant à la profession d'ingénierie et devraient placer un accent sur le processus de design. Ce dernier devrait être privilégié, car les problèmes abordés pendant le processus de design proviennent de la vie courante et demandent que les élèves utilisent leurs connaissances antérieures en STIM et diverses autres disciplines. Les 
formations devraient offrir des occasions pour que les enseignants puissent, eux-mêmes, essayer de résoudre un problème par l'entremise du processus de design et devraient permettre du temps de collaboration entre collègues pour concevoir des activités d'apprentissage du génie.

Il ne s'agit pas d'ajouter des leçons pour incorporer le génie, mais de changer la façon d'aborder les concepts scientifiques et les processus mathématiques. Au lieu d'un enseignement séparé en disciplines, le processus de design permet un apprentissage transdisciplinaire des STIM où les élèves tentent de résoudre des problèmes réels de la société. De fait, les problèmes impliquant l'environnement ou la santé publique intéressent davantage les filles [27], alors les activités du génie pourraient inciter les filles à se lancer dans des études en ingénierie.

En guise de conclusion, nous offrons des conseils au personnel enseignant. Nous recommandons qu'il s'informe davantage de la profession d'ingénierie et du processus de design, et qu'il incorpore ce processus dans les cours de sciences et des mathématiques afin d'entreprendre un enseignement transdisciplinaire des STIM. Enfin, nous conseillons au personnel enseignant de prendre un moment pour se regarder dans le miroir et de se poser la question : comment puis-je me rattraper pour engager des jeunes en génie?

\section{Références}

[1] Anderson, L.S., Gilbridge, K.A. et Bajaj, N. "Discover engineering follow-up surveys: Assessment/Evaluation of recruitment programs." Proceedings of the 2005 WEPAN/NAMEPA Joint Conference, Las Vegas, NV, 2005. Récupéré [le 9 janvier 2018] du site : https://www.researchgate.net/publication/229002227

Discover_Engineering_Followup_Surveys_Assessmentevaluation_of_recruitment_pr ograms

[2] Banilower, E. R., Smith, P. S., Weiss, I. R., Malzahn, K. A., Campbell, K. M., et Weis, A. M. Report of the 2012 National Survey of Science and Mathematics Education. Chapel Hill, NC: Horizon Research, Inc., 2013. Récupéré [le 4 février 2018] du site : http://www.horizon-research.com/2012nssme/wpcontent/uploads/2013/02/2012-NSSME-FullReport1.pdf

[3] Bell, D. "The reality of STEM education, a design and technology teachers' perceptions: a phenomenographic study." International Journal of Technology and Design Education, vol. 26, no. 1, 2016, pp. 61-79.

[4] Boesdorfer, S.B. "High School Chemistry Teachers' Views of Engineering Inclusion Before and After a Professional Development Program." Center for Educational Transformation, vol. 2, no. 1, 2017, pp. 16. Récupéré [le 4 février 2018] du site: https://cet.uni.edu/sites/default/files/boesdorfer_brief_ $\underline{0 . p d f}$

[5] Bouchard, J. Étude de la perception de la compétence d'élèves du secondaire à l'égard d'activités des cours de sciences physiques 416, 1997. Récupéré [le 15 novembre 2017] du site http://www.collectionscanada.gc.ca/obj/s4/f2/dsk2/ftp 03/MQ26547.pdf

[6] Bouchillon, E. What is STEM Education? Definition, Importance \& Standards, n.d. Récupéré [le 28 avril 2018] du site : https://study.com/academy/lesson/what-is-stemeducation-definition-importance-standards.html

[7] Brophy, S., Klein, S., Portsmore, M. et Rogers, C. "Advancing Engineering Education in P-12 Classrooms." Journal of Engineering Education, 2008, pp. 369-387. Récupéré [le 10 décembre 2017] $\mathrm{du}$ https://pdfs.semanticscholar.org/e151/9779d42eec0b3 768a758e71da85be18db3cc.pdf

[8] Brown, R., Brown, J, Reardon, K. et Merrill, C. "Understanding STEM: Current Perceptions." Technology and Engineering Teacher, 2011, pp. 5-9.

[9] Bull, G., Knezek, G. et Gibson, D. "Editorial: A Rationale for Incorporating Engineering Education Into the Teacher Education Curriculum." Contemporary Issues in Technology and Teacher Education Journal, 2016. Récupéré [le 22 novembre 2017] du site : http://www.citejournal.org/volume9/issue-3-09/editorial/editorial-a-rationale-forincorporating-engineering-education-into-the-teachereducation-curriculum/

[10] Bybee, R.W. "Advancing STEM Education: A 2020 Vision.” Technology and Engineering Teacher, 2010, pp. 30-35.

[11] Canada 2067. (2017). What is Canada 2067?, 2017. Récupéré [le 28 octobre 2017] du site : https://canada2067.ca/fr/a-propos/

[12] Carberry, A.R., Lee, H.S. et Ohland, M.W. "Measuring engineering design self-efficacy." Journal of Engineering Education, vol. 99, no. 1, 2010, pp. 71-79. Récupéré [le 15 juillet 2018] du site : https://ceeo.tufts.edu/documents/journal/carberry_lee_ ohland.pdf

[13] Chang, R. "Research: Boys Say They're More Likely to Pursue STEM Careers Than Girls." The Journal, 2017 Récupéré [le 28 avril 2018] du site : https://thejournal.com/articles/2017/06/20/researchboys-say-theyre-more-likely-to-pursue-stem-careersthan-girls.aspx

[14] Corbeil, A. Une analyse motivationnelle des intentions de carrière en contexte de PME, 2010. Récupéré [le 8 décembre 2017] du site : http://depote.uqtr.ca/1992/1/030141459.pdf

[15] Council of Ontario Directors of Education. Compétences mondiales du $21^{e}$ siècle - ébauche de l'Ontario, 2015. Récupéré [le 24 avril 2018] du site : http://www.ontariodirectors.ca/CODETLF/docs/tel/21_century_Fr_appendixC_only.pdf

[16] Creswell, J.W. Qualitative inquiry and research design: Choosing among five approaches. Sage publications, 1998. 
[17] DeCoito, I. "STEM Education in Canada: A Knowledge Synthesis". Canadian Journal of Science, Mathematics and Technology Education, vol. 16, no. 2, 2016, pp. 114-128.

[18] Ellis, C. Adams, T.E. et Bochner, A.P. "Autoethnography: An Overview." Forum Qualitative Sozialforschung / Forum: Qualitative Social Research, vol., 12, no. 1, Art. 10, 2011 Récupéré [le 13 janvier 2019] du site: http://nbnresolving.de/urn:nbn:de:0114-fqs1101108.

[19] Ferreira, M. "Ameliorating equity in science, mathematics, and engineering: A case study of an after-school science program." Equity \& Excellence in Education, vol. 35, no. 1, 2002, pp. 43-49.

[20] Gouvernement du Canada. Maximizing Canada's Engagement in the Global Knowledge-Based Economy: 2017 and Beyond, 2017. Récupéré [le 20 octobre 2017] du site : http://www.horizons.gc.ca/eng/content/maximizingcanada\%E2\%80\%99s-engagement-global-knowledgebased-economy-2017-and-beyond\%C2\%A0

[21] Hacioglu, Y., Yamak, H. et Kavak, N. "The Opinions of Prospective Science Teachers Regarding STEM Education: The Engineering Design Based Science Education." GEFAS\GUJGEF, vol. 37, no. 2, 2017, pp. 649-684.

[22] Hammack, R.J. Elementary Teachers' Perceptions of Engineering, Engineering Design, And Their Abilities to Teach Engineering: A mixed methods study (Order No. 10188936). Available from ProQuest Dissertations \& Theses Global. (1854867429), 2016.

[23] Hammack, R. et Ivey, T. "Examining Elementary Teachers' Engineering Self-Efficacy and Engineering Teacher Efficacy." School Science and Mathematics, vol. 117, no. 1-2, 2017, pp. 52-62.

[24] Hammack, R., Ivey, T.A., Utley, J. et High, K.A. "Effect of an Engineering Camp on Students' Perceptions of Engineering and Technology." Journal of Pre-College Engineering Education Research, vol. 5, no. 2, 2015, pp. 10-21. Récupéré [le 9 décembre 2017] du site : http://docs.lib.purdue.edu/cgi/viewcontent.cgi?article $=$ $1102 \&$ context=jpeer

[25] Hsu, M., Purzer, S. et Cardella, M. E. "Elementary teachers' views about teaching design, engineering, and technology." Journal of Pre-College Engineering Education Research, vol. 1, no. 2, 9, 2011.

[26] Idaho Department of Education. What is STEM Education? 2013. Récupéré [le 28 avril 2018] du site : https://www.education.com/reference/article/whatstem-education-science-technology/

[27] Ing, M., Aschbacher, P.R. et Tsai, S.M. "Gender Differences in the Consistency of Middle School Students' Interest in Engineering and Science Careers". Journal of Pre-College Engineering Education Research, vol. 4, no. 2, 2014, pp. 1-10. Récupéré [le 20 avril 2018] du site : https://docs.lib.purdue.edu/cgi/viewcontent.cgi?article $=1090 \&$ context=jpeer

[28] Innovation, Sciences et Développement économique Canada. On n'est jamais trop jeune pour être un innovateur, Gouvernement du Canada, 2017.
Récupéré [le 26 octobre 2017] du site : https://www.canada.ca/fr/innovation-sciencesdeveloppementeconomique/nouvelles/2017/05/on n est jamais tropj eunepouretreuninnovateur.html

[29] Kelley, T.R. et Knowles, J.G. "A conceptual framework for integrated STEM education." International Journal of STEM Education, vol. 3, no. 11, 2016. Récupéré [le 13 janvier 2019] du site: https://stemeducationjournal.springeropen.com/track/p df/10.1186/s40594-016-0046-z

[30] Khandani, S. "Engineering Design Process". Education Transfer Plan, 2005. Récupéré [le 11 novembre 2018] du site : https://www.saylor.org/site/wpcontent/uploads/2012/09/ME101-4.1-EngineeringDesign-Process.pdf

[31] Kimmel, H., Carpinelli, J., Burr-Alexander, L. et Rockland, R. Bringing Engineering Into K-12 Schools: A Problem Looking for Sultions? Papier présenté au 2006 Annual Conference \& Exposition, Chicago, Illinois, 2006. Récupéré [le 13 janvier 2018] du site : https://peer.asee.org/181

[32] Knapp, M. C. "An autoethnography of a (reluctant) teacher leader". Journal of Mathematics Behavior, vol. 46, 2017, pp. 251-266. Récupéré [le 2 mars 2019] du site : https://journals-scholarsportalinfo.proxy.bib.uottawa.ca/pdf/07323123/v46icomplete /251_aaoatl.xml

[33] Langdon, D., McKittrick, G, Beede, D., Khan, B. et Doms, M. "STEM: Good Jobs Now and for the Future." U.S. Department of Commerce. Economics and Statistics Administration, 2011. Récupéré [le 15 octobre 2017] du site : http://www.esa.doc.gov/sites/default/files/stemfinalyju ly14_1.pdf

[34] Lent, R. W. "Une conception sociale cognitive de l'orientation scolaire et professionnelle : considérations théoriques et pratiques." L'orientation scolaire et professionnelle, vol. 37, no. 1, 2008. Récupéré [le 8 décembre 2017] du site : http://journals.openedition.org/osp/1597\#tocto2n2

[35] Lent, R. W., Brown, S. D. et Hackett, G. "Toward a unifying social cognitive theory of career and academic interest, choice, and performance." Journal of Vocational Behavior, vol. 45, 1994, pp. 79-122. Récupéré [le 8 décembre 2017] du site : https://searchproquest-

com.proxy.bib.uottawa.ca/docview/62824934?account id=14701

[36] Lent, R.W., Brown, S.D. et Hackett, G. Social cognitive career theory. Career Choice and Development, $4^{\mathrm{e}}$ édition, 2002, pp. 750-754.

[37] Loisy, C. et Carosin, E. "Concevoir et accompagner le développement du pouvoir d'agir des adolescent.e.s dans leur orientation." Orientation Scolaire et Professionnelle, vol. 46, no. 1, 2017, pp. 61-87.

[38] Madara, D.S. et Namango, S. "Perceptions of Female High School Students on Engineering." Journal of Education and Practice, vol. 7, no. 25, 2016, pp. 6382. 
[39] Meyrick, K.M. "How STEM Education Improves Student Learning." Meridian K-12 School Scompter Technologies Journal, vol. 14, no. 1, 2011. Récupéré

[40] Ministère de l'Éducation. The Ontario Curriculum Grades 1-8- Science and Technology, 2007. Récupéré [le 27 octobre 2017] du site : http://www.edu.gov.on.ca/eng/curriculum/elementary/ scientec18currb.pdf

[41] Ministère de l'Éducation de l'Ontario. Nouvelle vision de l'éducation, 2019. Récupéré [le 3 avril 2019] du site https://efis.fma.csc.gov.on.ca/faab/Memos/B2019/B08 FR.pdf

[42] National Academy of Engineering and National Research Council. Engineering in K-12 Education: Understanding the Status and Improving the Prospects. Washington, DC: The National Academies Press, 2009.

[43] National Science Board. Science and engineering indicators 2008. Arlington, VA: National Science Foundation (NSB-08-1), 2008. Récupéré [le 13 janvier 2018] du site : https://wayback.archiveit.org/5902/20160210222516/http://www.nsf.gov/stati stics/seind08/pdfstart.htm

[44] Sanders, M. "STEM, STEM Education, STEMmania". The Technology Teacher, 2009, pp. 20-26.

[45] Thompson, C. "8 jobs every company will be hiring for by 2020." World Economic Forum, 2016. Récupéré [le 14 octobre 2017] du site : https://www.weforum.org/agenda/2016/01/8-jobsevery-company-will-be-hiring-for-by-2020/

[46] Turkle, S. A Passion for Objects. The Chronicle of Higher Education, 2008 Récupéré [le 12 janvier 2018] du site

http://web.mit.edu/sturkle/www/pdfsforstwebpage/ST Passion\%20for\%20Objects.pdf

[47] UNESCO. Engineering: Issues Challenges and Opportunities for Development, 7, place de Fontenoy, 75352 Paris 07 SP, France, ISBN 978-92-3-104156-3, [le 22 novembre 2017] du site : http://www.smartmovesplayplace.com/newsarticle.php?id=1

2010. Récupéré [le 13 janvier 2018] du site : http://unesdoc.unesco.org/images/0018/001897/18975 3e.pdf

[48] UNESCO. UNESCO Science Report : Towards 2030, 2015. Récupéré [le 28 octobre 2017] du site: http://unesdoc.unesco.org/images/0023/002354/23540 6e.pdf

[49] UNESCO. Cracking the Code: Girls' and women's education in science, technology, engineering and mathematics (STEM), 2017. Récupéré [le 4 décembre 2017] du site : http://unesdoc.unesco.org/images/0025/002534/25347 9E.pdf

[50] Viau, R. "La motivation: condition au Plaisir d'apprendre et d'enseigner en contexte scolaire." $3^{e}$ congrès des chercheurs en Éducation. Bruxelles, 2004. Récupéré [le 15 novembre 2017] du site: https://projetadef.files.wordpress.com/2011/12/la mot ivation.pdf

[51] Vilorio, D. "STEM 101: Intro to tomorrow's jobs." Occupational Outlook Quaterly, 2014. Récupéré [le 15 octobre 2017] du site https://www.bls.gov/careeroutlook/2014/spring/art01. pdf

[52] Wiswall, M., Stiefel, L., Schwartz, A. E., et Boccardo, J. "Does attending a STEM high school improve student performance?" Evidence from New York City. Economics of Education Review, vol. 40, 2014, pp. 93-105. DOI: $10.1016 /$ j.econedurev.2014.01.005

[53] Wolcott, H.F. The art of fieldwork. Rowman Altamira, 2005.

[54] Yoon, S. Y., Diefes-Dux, H. et Strobel, J. "First-Year Effects Of An Engineering Professional Development Program On Elementary Teachers." American Journal of Engineering Education, vol. 4, no. 1, 2013, pp.

67-84. 